\title{
Early Post-Stroke Electroacupuncture Promotes Motor Function Recovery in Post-Ischemic Rats by Increasing the Blood and Brain Irisin
}

This article was published in the following Dove Press journal: Neuropsychiatric Disease and Treatment

\author{
Li Liu'l,* \\ Qun Zhangl,* \\ Mingyue $\mathrm{Li}^{2}$ \\ Nianhong Wang' \\ $\mathrm{Ce} \mathrm{Li}{ }^{1}$ \\ Di Song ${ }^{3}$ \\ Xueyan Shen' \\ Lu Luo' \\ Yunhui Fan' \\ Hongyu Xie' \\ Yi Wu'
}

'Department of Rehabilitation Medicine, Huashan Hospital, Fudan University,

Shanghai, People's Republic of China;

${ }^{2}$ Department of Rehabilitation Medicine,

The Third Affiliated Hospital, Sun Yat-sen

University, Guangzhou, People's Republic

of China; ${ }^{3}$ Department of Rehabilitation

Medicine, The Affiliated Sixth People's

Hospital of Shanghai Jiaotong University,

Shanghai, People's Republic of China

*These authors contributed equally to this work
Correspondence: Hongyu Xie; Yi Wu Department of Rehabilitation Medicine, Huashan Hospital, Fudan University, 12 Wulumuqi Middle Road, Shanghai, 200040, People's Republic of China Email huangdaitian@I63.com; 16I II220064@fudan.edu.cn
Objective: Recent studies have shown that irisin, a novel peptide hormone derived from muscles, could be used as a potential therapeutic drug against ischemic stroke. Moreover, electroacupuncture (EA) is widely used in the treatment of ischemic stroke. Yet, whether irisin is involved in the EA neuroprotection remains unclear. The following study investigated the association between serum and peri-lesional cortex irisin and EA-induced post-stroke motor recovery in rats.

Methods: The middle cerebral artery occlusion (MCAO) method was used to induce ischemic stroke in rats. Rats were randomly divided into two groups: a middle cerebral artery occlusion (MCAO) group (MCAO rats without treatment) and an electroacupuncture (EA) group (MCAO rats treated with EA). On the 3rd day post-stroke, infarct volume, behavioral deficits, surviving neurons, irisin protein expression in peri-infarction cortex, muscle tissue, and serum were evaluated to identify the neuroprotective of EA in acute ischemic stroke.

Results: Compared with the MCAO group, the EA group showed better behavioral performance, a smaller cerebral infarct volume, more surviving neurons, and a significant increase in irisin expression in the peri-infarction cortex and serum $(p<0.05)$. However, no difference in irisin expression in the muscle tissue was found between the MCAO group and the EA group $(p>0.05)$. Conclusion: EA promotes motor function recovery, reduces the volume of cerebral infarction, and alleviates neuronal death following ischemic stroke by enhancing the expression of irisin in both the blood and peri-lesional cortex.

Keywords: ischemic stroke, electroacupuncture, irisin

\section{Introduction}

Stroke is a leading cause of morbidity and mortality worldwide. ${ }^{1}$ Acute thrombolytic therapy is the most effective treatment for patients suffering from ischemic stroke. $^{2}$ This kind of therapy is usually given within 4-6 hours after onset of an ischemic stroke, while its effectiveness beyond this time frame ( $>6 \mathrm{~h})$ still remains debatable. Ischemic stroke is a complex inflammatory cascade. Cerebral ischemia can cause acute neuroinflammation and neuronal injury. In the subacute and chronic phases, cerebral ischemic inflammation can lead to glial cell proliferation, neuronal apoptosis, and brain tissue atrophy. These processes are associated with the impairment of nerve function. ${ }^{3}$

Recent studies have shown that irisin, cleaved from precursor fibronectin type III domain-containing protein 5 (FNDC5), could be used as a potential therapeutic drug against ischemic stroke. Emerging evidence has indicated that the serum levels 
of irisin could predict outcomes of patients with ischemic stroke. ${ }^{4}$ In addition, irisin can also promote neuronal cell survival $^{5}$ and inhibit post-stroke inflammation, reducing cerebral infarction volume in MCAO models. ${ }^{6}$ Since irisin is released from skeletal muscle during exercise, ${ }^{7}$ any treatment that stimulates muscle contraction may be used as a potential therapy to increase the irisin post-stroke. However, it is still unclear whether peripheral or central irisin mediates the effects in the brain.

Previous studies have found that early EA can improve neurobehavioural functional recovery and reduce infarct volume by inhibiting the brain inflammation, ${ }^{8}$ oxidative stress, ${ }^{9}$ and promoting angiogenesis. ${ }^{10}$ Moreover, EA stimulation at peripheral limbs, for instance, at Zusanli (ST36) and Quchi (LI11) points, could also increase the serum factors, like brain-derived neurotrophic factor (BDNF) and glial cell line-derived neurotrophic factor (GDNF). ${ }^{11}$ Recent studies found that long-term EA stimulation promotes the expression of FNDC5 in skeletal muscle of diet-induced obesity rats. ${ }^{12}$ However, whether irisin is involved in EA neuroprotection remains unclear.

The aim of this study was to investigate the efficacy of EA stimulation at Zusanli (ST36) and Quchi (LI11) points on post-stroke recovery. In order to further explore the early EA neuroprotection mechanism, we analyzed different irisin concentrations in the muscle, serum, and pei-infarct cortex on the 3rd day of the post-ischemic stroke. We hypothesized that EA stimulates muscle contraction, increasing the skeletal muscle-originated irisin in either blood or the peri-lesional cerebral cortex, thus resulting in an infarct reduction (Figure 1).

\section{Materials and Methods}

\section{Animals}

All experimental procedures were carried out in accordance with the Guidelines for Animal Experimentation of Fudan University and were approved by the Ethics Committee for Animal Experimentation (No: 2019Huashan Hospital JS-139). Male Sprague-Dawley rats $(250-280 \mathrm{~g})$ of clean grade were obtained from the Experimental Animal Centre of Fudan University. The SD rats were housed in a $12 \mathrm{~h}$ light-dark cycle at a room temperature of $20 \sim 25^{\circ} \mathrm{C}$, with a humidity of $60 \sim 70 \%$, and were allowed free access to food and water for at least 1 week.

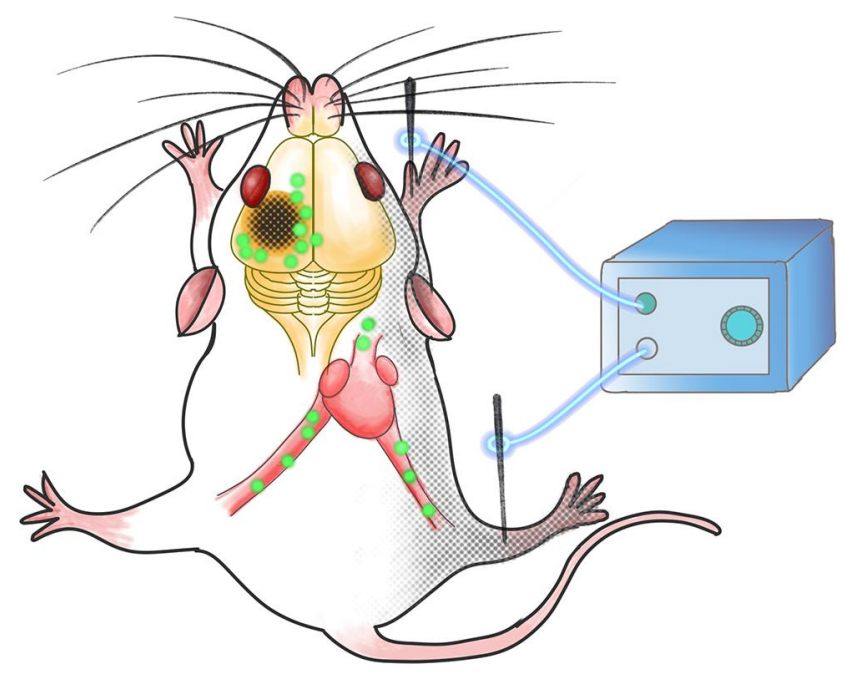

Figure I The schematic of EA stimulation efficacy on irisin expression. EA stimulation at Zusanli (ST36) and Quchi (LIII) points on the paralyzed limbs promotes the poststroke recovery through both the peri-lesional cortex and serum irisin elevation.

\section{Middle Cerebral Artery Occlusion (MCAO) Model}

The middle cerebral artery occlusion (MCAO) model was induced in male SD rats, as previously described. ${ }^{13}$ Rats were anesthetized with $3 \%$ pentobarbital sodium $(30 \mathrm{mg} / \mathrm{kg})$. A monofilament (2636-A5, Beijing Cinontech Co., Ltd., Beijing, China) with a silicone-coated tip was used to obstruct the middle cerebral artery (MCA) through the left external carotid artery and the internal carotid artery; it was left in place for 90 minutes. The rats were then randomly and evenly divided into two groups: a middle cerebral artery occlusion (MCAO) group (MCAO rats without treatment) and an electroacupuncture (EA) group (MCAO rats treated with EA).

\section{Electroacupuncture (EA) Treatment}

The Quchi (LI11) and Zusanli (ST36) acupoints on the paralyzed right limbs were stimulated during MCAO surgery. An intensity of $1 \sim 2 \mathrm{~mA}$ and an intermittent wave frequency of $2 \sim 10 \mathrm{~Hz}$ for $90 \mathrm{~min}$ were provided with the Hwatuo electronic acupuncture treatment instrument (model No. SDZ-V, Suzhou Medical Appliances Co., Ltd., Suzhou, China). The rats were treated with electroacupuncture under anesthesia. The body temperature was maintained at $37 \pm 0.5^{\circ} \mathrm{C}$.

\section{Modified Neurological Severity Scores ( $\mathrm{mNSS}$ )}

The modified Neurological Severity Scores (mNSS) were used to evaluate rats' motor, sensory, and reflex functions, 
respectively. A higher score represented serious nerve injury. mNSS evaluation was performed on the third day after surgery. ${ }^{14}$

\section{Ladder Rung Walking}

The ladder rung walking was used to assess the number of errors in animals' anterior and posterior limbs. The apparatus consisted of two plexiglass walls $(100 \mathrm{~cm} \times$ $20 \mathrm{~cm} \times 0.8 \mathrm{~cm})$ and metal rungs. The width of the alley was $8 \mathrm{~cm}$ apart to allow for the passage of a rat. On the third day after surgery, animals were challenged to cross the ladder; a regular pattern of the rungs (spaced at $2 \mathrm{~cm}$ intervals) allowed for the testing of animals. A camera was positioned at a slight ventral angle, which allowed shooting of foot positions that were then used for further analysis. $^{15}$

\section{Rotarod Test}

Motor coordination in rats was evaluated using the rotarod test. The rotarod apparatus consists of a bench pad and a rod, which set the acceleration to 4-40 revolutions per minute (rpm). All the rats were trained for three consecutive days before surgery. Each rat was trained for 5 min and underwent 3 trials per day; rats were allowed to rest for a $15 \mathrm{~min}$ inter-trial interval so as to avoid fatigue. The test was performed on the 3rd day post-ischemic stroke. Three rats could be simultaneously tested. The rotarod's time was recorded when the rat fell off the rod, and the meantime was calculated from three trials for each rat. $^{16}$

\section{Cerebral Infarct Volume}

The cerebral infarct volume was measured on the 3rd day post-ischemic stroke. The rats were anesthetized (i.p) with $3 \%$ pentobarbital sodium $(30 \mathrm{mg} / \mathrm{kg})$, after which the anesthetized animals were sacrificed by cervical dislocation. Brain samples were then collected, kept at $-80^{\circ} \mathrm{C}$ for $3 \mathrm{~min}$, cut into 6 coronal 2-mm-thick sections, incubated in $2 \%$ 2,3,5-triphenyltetrazolium chloride (TTC) at $37^{\circ} \mathrm{C}$ for $30 \mathrm{~min}$ in the dark, followed by scanning with a digital camera. The white color regions were defined as infarct areas. The volume ratio of cerebral infraction $=\{$ [total infarct volume-(left hemisphere volume-right hemisphere volume)]/right hemisphere volume $\} \times 100 \% .{ }^{17}$ An independent investigator, who was blinded to the grouping, performed the measurements.

\section{Western Blot}

The protein expression of FNCD5 was performed by Western blot on the 3rd day post-ischemic stroke. Periinfarction cortex tissues and muscle tissue samples were homogenized in radioimmunoprecipitation assay (RIPA) buffer. The homogenate was centrifuged in a centrifugal machine for $15 \mathrm{~min}$ at $12,000 \mathrm{rpm}$, after which the supernatant samples were collected in a new $1.5 \mathrm{~mL}$ tube. Protein concentrations were determined with a bicinchoninic acid (BCA) protein assay kit (Melone Pharmaceutical Co., Ltd., Dalian, China). The lysates were separated using 12\% sodium dodecyl sulfate-polyacrylamide (SDS-PAGE) gels and transferred onto polyvinylidene difluoride (PVDF) membranes. The membranes were blocked with $5 \%$ nonfat milk in TBST for $1 \mathrm{~h}$ at room temperature and then incubated overnight at $4^{\circ} \mathrm{C}$ with the following primary antibodies: FNCD5 (1:1000, ab174833, Abcam, USA) and $\beta$-actin (1:1000, MA5-15,739, Invitrogen, United States). The next day, the membrane was washed in TBST and incubated for $1 \mathrm{~h}$ at room temperature with HRP-conjugated goat anti-rabbit antibody and goat anti-mouse antibody.

\section{Measurement of Serum Irisin Levels}

On the 3rd day post-ischemic stroke, $1 \mathrm{~mL}$ blood samples were drawn from the rat hearts. The samples were centrifuged at $4{ }^{\circ} \mathrm{C}$ and $4000 \mathrm{rpm}$ for $15 \mathrm{~min}$, after which the supernatants were collected in a new tube. The irisin levels in the serum were measured using a commercial ELISA kit. The assay was conducted according to the manufacturer's instructions. The absorbance was measured at $450 \mathrm{~nm}$, and the analysis of interest was quantified using a standard curve.

\section{Nissl Staining}

Nissl staining was performed on the 3rd day postischemic stroke. Five rats in each group were randomly selected for abdominal anesthesia. Their brains were fixed in $4 \%$ paraformaldehyde, dehydrated, embedded in paraffin, and sectioned. Then, samples were rinsed in distilled water for 3 times ( 5 min each time) and placed in $0.5 \%$ toluidine blue for $1 \mathrm{~min}$ at room temperature. Stained sections were then fixed by serial dehydration in alcohol and xylene before coverslipping with neutral gum. The images were observed and photographed under a light microscope; cumulative optical 
density values of Nissl were analyzed with Image-Pro Plus 6.01 Image analysis software. The quantity of Nissl bodies in normal neurons is abundant. When neurons are damaged, the Nissl bodies decrease, disintegrate, or disappear.

\section{TUNEL Staining}

Paraffin sections were performed on the brain after perfusion to prepare coronal sections (4um). Each sample was cut into 5 pieces. The dewaxed and hydrated samples were then rinsed twice with phosphate buffer and stained using a TUNEL apoptosis kit (G1501, Wuhan Servicebio technology Co., Ltd., Wuhan, China). The apoptotic cells were observed under a fluorescence microscope after sealing. The cells with apoptosis were cultured in green.

\section{Statistical Analysis}

GraphPad Prism 8.0 was used to perform all statistical analyses. All values were presented as mean \pm SEM by the unpaired $t$-test. Normal distribution was confirmed by the Shapiro-Wilk test. A $p$-value $<0.05$ was used to indicate a statistical significance.

\section{Results}

\section{EA Promotes the Ischemic Motor Function in Rats}

To investigate the neuroprotective effect of EA on MCAO, we assessed the neurobehavioural function on the 3rd day post-ischemic stroke. The results revealed that the mNSS score in MCAO group was increased, while it was decreased in the EA group ( $7.83 \pm 0.16$ vs. $6.83 \pm 0.29$; $p<0.05$ ) (Figure 2A). Moreover, rats in the MCAO spent less time on the rotarod than rats treated with EA (84.45 $\pm 13.35 \mathrm{~s}$ vs. $129.0 \pm 14.70 \mathrm{~s})$, and the EA group achieved better test performance $(p<0.05)$ (Figure $2 \mathrm{~B})$. In addition, the number of slips in the MCAO group was $72.31 \pm 3.33$, while in the EA group, it was $56.31 \pm 3.50 \quad(p<0.05)$ (Figure 2C).

\section{EA Reduces the Infarction Volume}

Infarction volume was assessed by TTC staining on the 3rd day post-ischemic stroke. The white infarct area was presented in the left hemisphere, as shown in Figure 3A. Compared with the MCAO group, the EA group infarction volume was reduced $(50.01 \pm 4.08 \%$ vs. $36.88 \pm 3.95 \%$; $p<0.05$ ) (Figure 3B).

\section{EA Increases the Concentrations of Irisin in Serum, Peri-Lesional Cortex Irisin Expression but Had No Effect on Muscle Irisin After 3-Day Post-Stroke}

To evaluate the effect of EA on the concentration of irisin in the serum on the 3rd day post-ischemic stroke, ELISA test was used to assess the concentrations of irisin. A significant increase in the concentrations of irisin was found in the EA group $(64.04 \pm 3.82)$ compared to the MCAO group $(27.25 \pm 1.44, p<0.05)$ (Figure 3C).

Western blot analysis is shown in Figure 4A and B. Compared with the MCAO group, EA promoted irisin expression in the peri-lesional cortex; the expression of irisin was $0.17 \pm 0.02$ in the MCAO group and $0.28 \pm 0.04$ in the EA group $(p<0.05)$ (Figure $4 \mathrm{C})$. However, no difference in irisin expression in the muscle tissue was found in the MCAO group and the EA group $(0.92 \pm 0.18$ vs. 0.94 $\pm 0.28 ; p>0.05$ ) (Figure 4D).

\section{EA Reduced Neuropathic Damage After Cerebral Ischemia}

Nissl staining and TUNEL staining were used to assess the effect of EA on neurons on the 3rd day post-ischemic stroke (Figure 5A and B). Nissl staining showed a large number of death cells in the MCAO group compared to the EA group, which showed an increased number of surviving neurons in the EA group $(16.62 \pm 1.07$ vs. $25.36 \pm 0.92$, $p<0.05$ ) (Figure 5C). Furthermore, the TUNEL assay indicated that TUNEL-positive neurons were significantly higher in the MCAO group than the EA group (100.80 \pm 5.16 vs. $70.16 \pm 4.62, p<0.05$ ) (Figure $5 \mathrm{D}$ ).

\section{Discussion}

This study found that EA could promote post-stroke motor function recovery and reduce infarct volume, which may be related to the improvement in irisin expression in the peri-infarct cortex and serum. Irisin is the most recently discovered and isolated hormone released from muscles into the circulation after physical exercise, ${ }^{18}$ which is found in the brain, heart, kidney, liver, lung, and adipose tissue. ${ }^{19,20}$ Huh et al discovered that muscle tissue and circulating irisin levels significantly increased thirty minutes after acute exercise, ${ }^{21}$ while endurance exercise or long-term exercise increased the expression of FNDC5 in muscle tissue or brain tissue, like the hippocampus. ${ }^{22,23}$ A previous study showed that exercise could promote the 

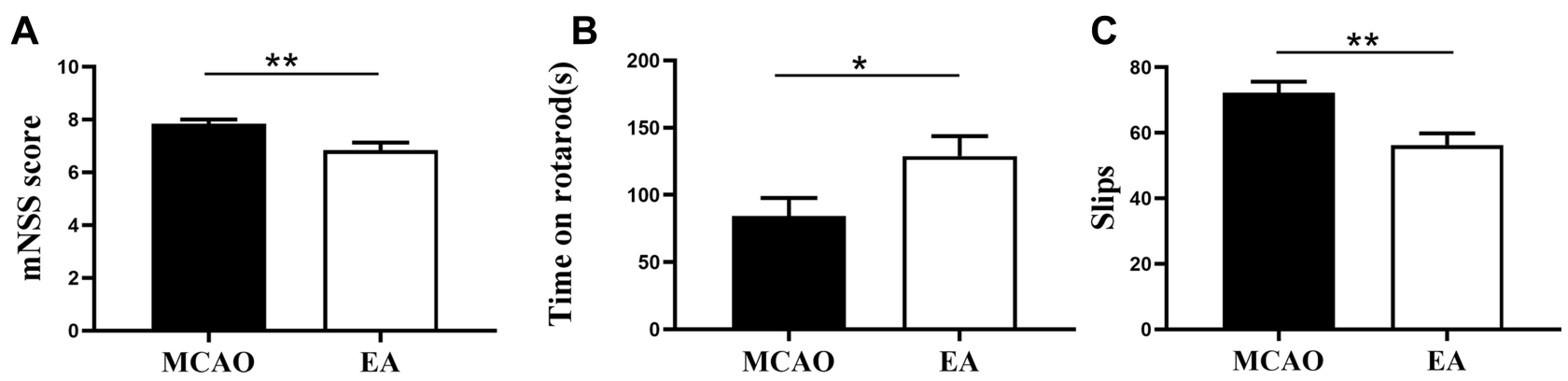

Figure 2 Effects of EA on motor recovery on the 3rd day post-ischemic stroke. (A) The mNSS score to assess the motor and sensory function recovery following ischemic stroke. (B) The Rotarod test to value the motor coordination recovery. (C) The Ladder rung walking test to assess the number of errors of the anterior and posterior limbs of animals. ( $n=12$ in MCAO group, $n=13$ in EA group). ${ }^{*} p<0.05$, ${ }^{*} p<0.01$ compared to MCAO group.

A

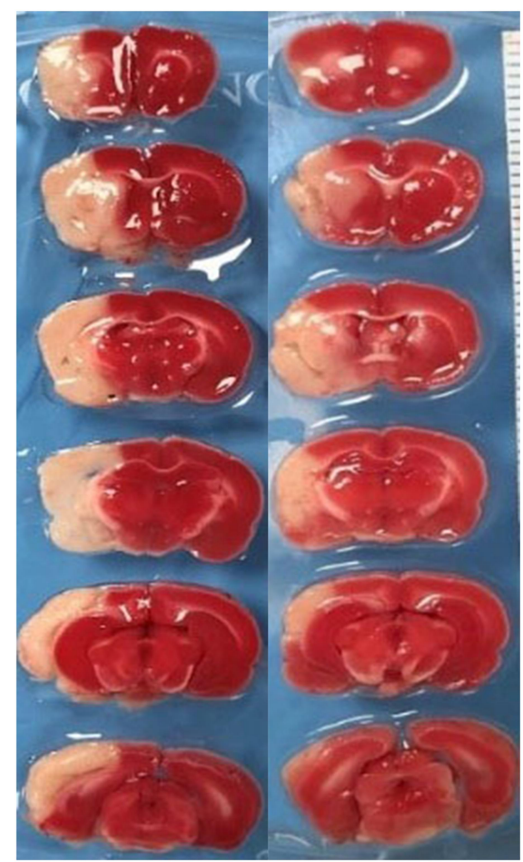

MCAO

EA
B
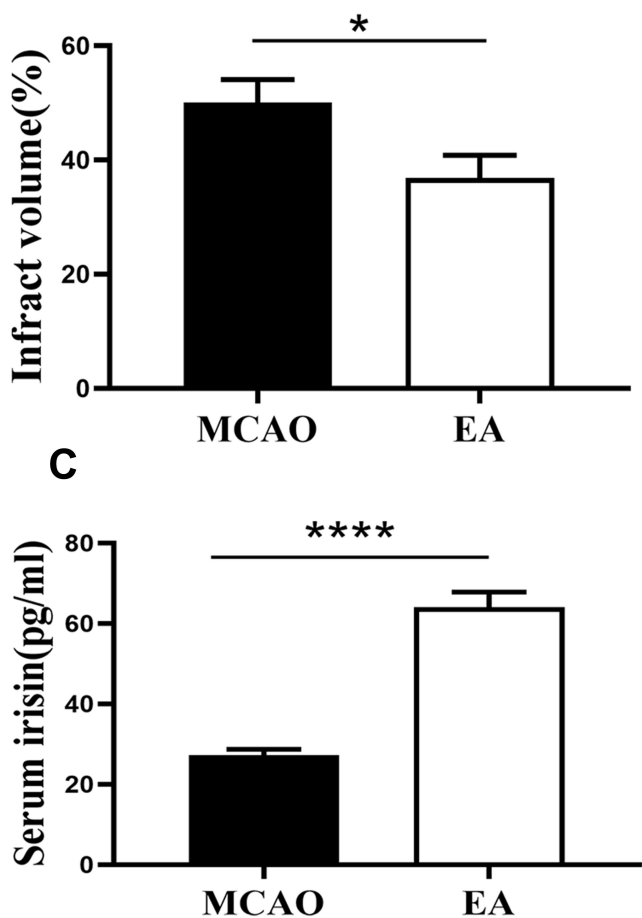

Figure 3 The infarct volume and concentrations of irisin in serum on the 3 rd day post-ischemic stroke. (A) The infarct volume was valued by TTC staining, the white color region is cerebral infraction, and the red color region is normal brain tissue. (B) The percentage of infarct volume ( $n=12$ in MCAO group, $n=13$ in EA group). (C) Quantification of concentrations of irisin in serum $(n=5)$. ${ }^{*} p<0.05, * * * * p<0.0001$ compared to MCAO group.

expression of irisin in the muscle of cerebral ischemia mice, although the concentration of irisin in the plasma and muscle decreased following cerebral ischemia. Physical exercise could protect against the ischemic by inducing irisin expression through activation of the Protein Kinase B (Akt) and extracellular signal-regulated kinase $(E R K 1 / 2){ }^{6}$ In this study, we also found an increased concentration of irisin in the serum and a smaller cerebral infarction volume in the EA group. In our study, on the 3rd day after EA stimulation, the expression of FNCD5 was enhanced in the peri-infarct cortex and serum, but not in the muscle tissue, which may be related to the short time of EA stimulation or different modes of intervention.

Electroacupuncture has shown therapeutic benefits in stroke patients and animal stroke models. ${ }^{24-27}$ Increasing evidence has shown that EA may affect the nerve conduction, endocrine hormone, and lymphatic circulation, thus promoting the recovery of the body function. ${ }^{28,29}$ EA stimulates local receptors of limbs; it urges the receptor 


\section{A MCAO EA}

FNCD5

$\beta$-actin

C

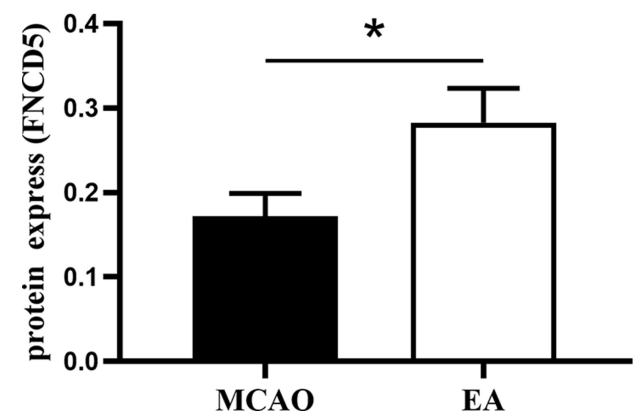

B MCAO EA

FNCD5

$\beta$-actin

D

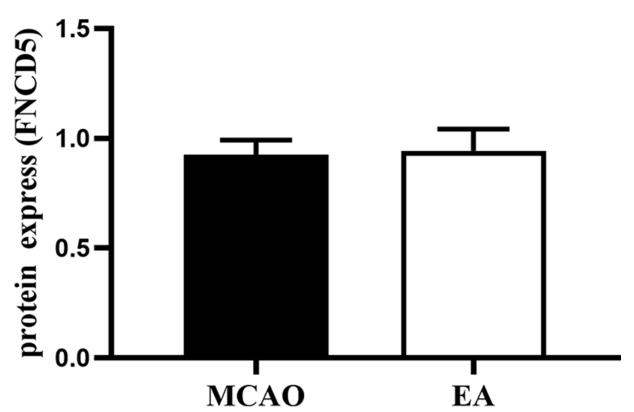

Figure 4 The expression of FNCD5 on the 3rd day post-ischemic stroke. (A) Western blot of FNCD5 expression in the peri-infarct cortex. (B) Western blot of FNCD5 expression in muscle tissue. (C, D) Quantitative analysis for the FNCD5 expression, respectively ( $n=6$ in MCAO group, $n=8$ in EA group). * $p<0.05$ compared to MCAO group.
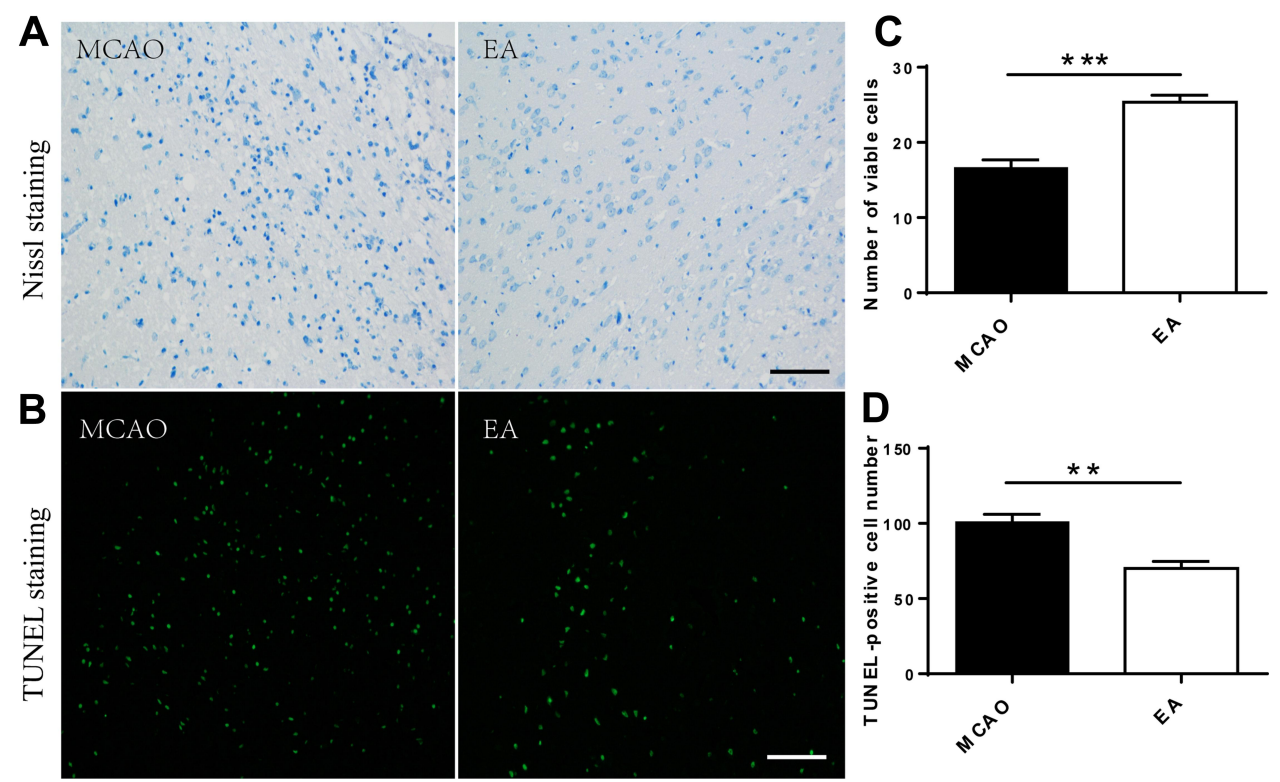

Figure 5 Surviving neurons count in the peri-lesional cerebral cortex on the 3rd day post-ischemic stroke. (A, B) Representative images of Nissl staining and TUNEL staining in different groups (scale bar $=100 \mu \mathrm{m}$ ). (C) Numerical analysis of Nissl-stained neurons. (D) Numerical analysis of TUNEL-positive neurons $(\mathrm{n}=5)$. ${ }^{*} p<0.0 \mathrm{I}$, $*_{* *} p<0.00$ I compared to MCAO group.

to distribute nerve impulses to the spinal cord, activates the uplink pathway to the brain, and induces the release of neurotransmitters so as to regulate the function of the brain and achieve the therapeutic effect. ${ }^{28}$ In our study, EA increased the irisin concentration in blood and periinfarction, while no significant change was observed in the muscle tissue. This may be because irisin, which originates from the muscles, was released into the blood circulation, thus resulting in the blood irisin enhancement. Moreover, several previous studies have indicated that FNDC5/irisin is highly expressed in skeletal muscle ${ }^{30}$ and in various regions of brain tissue, including cortical neurons, hippocampus, cerebellum, hypothalamus, and spinal cord. ${ }^{23,31}$ In this study, we found that irisin was 
more robustly increased in the brain after post-stroke electroacupuncture. These may be related to more neurogenesis in the perilesional cortex after electroacupuncture since FNDC5 was reported to be involved in the early regulation of neurogenesis. ${ }^{32}$ Therefore, it is interesting to confirm the source of the irisin, which may be responsible for the neurogenesis. In the future study, we plan to further explore the source of irisin in the brain and the nerve and blood irisin's respective role in EA-induced post-stroke recovery.

This study has several limitations. In our experiments, the control group received no intervention. In our next study, we plan to cut off the sciatic nerve for EA intervention to observe whether there would be any differences in results.

In conclusion, our research indicated that EA promotes motor function recovery and reduces cerebral infarction volume post-stroke. This may be related to neuronal activation that promotes increased expression of irisin protein in the peri-lesional cortex and serum, both of which are critical for cerebral neuroprotection.

\section{Acknowledgments}

The Natural Science Foundation of China (NSFC, nos. 81702218, 81672242, 81972141, 81972140, 82002392), the Key Projects of Shanghai Science and Technology on Biomedicine (nos.17411953900, 18401970200), Shanghai Municipal Key Clinical Specialty (nos.shslczdzk02702), and Shanghai Sailing Program (20YF1403500) supported this work.

\section{Disclosure}

The authors have no conflicts of interest to declare.

\section{References}

1. Xu L, Li Y, Fu Q, et al. Perillaldehyde attenuates cerebral ischemiareperfusion injury-triggered overexpression of inflammatory cytokines via modulating Akt/JNK pathway in the rat brain cortex. Biochem Biophys Res Commun. 2014;454(1):65-70. doi:10.1016/j. bbrc.2014.10.025

2. Hacke W, Kaste M, Bluhmki E, et al. Thrombolysis with alteplase 3 to 4.5 hours after acute ischemic stroke. $N$ Engl J Med. 2008;359 (13):1317-1329. doi:10.1056/NEJMoa0804656

3. Amani H, Habibey R, Shokri F, et al. Selenium nanoparticles for targeted stroke therapy through modulation of inflammatory and metabolic signaling. Sci Rep. 2019;9(1):6044. doi:10.1038/s41598-019-42633-9

4. Wu H, Guo P, Jin Z, et al. Serum levels of irisin predict short-term outcomes in ischemic stroke. Cytokine. 2019;122:154303. doi:10.1016/j.cyto.2018.02.017

5. Hosseini FS, Ghaedi K, Ghazvini ZF, et al. ERK1/2 is a key regulator of Fndc5 and PGC1alpha expression during neural differentiation of mESCs. Neuroscience. 2015;297:252-261. doi:10.1016/j.neurosci ence.2015.03.069
6. Li DJ, Li YH, Yuan HB, et al. The novel exercise-induced hormone irisin protects against neuronal injury via activation of the Akt and ERK1/2 signaling pathways and contributes to the neuroprotection of physical exercise in cerebral ischemia. Metabolism. 2017;68:31-42. doi:10.1016/j.metabol.2016.12.003

7. Novelle MG, Contreras C, Romero-Pico A, et al. Irisin, two years later. Int J Endocrinol. 2013;2013:746281. doi:10.1155/2013/746281

8. Zhan J, Qin W, Zhang Y, et al. Upregulation of neuronal zinc finger protein A20 expression is required for electroacupuncture to attenuate the cerebral inflammatory injury mediated by the nuclear factor-kB signaling pathway in cerebral ischemia/reperfusion rats. J Neuroinflammation. 2016;13(1):258. doi:10.1186/s12974-016-0731-3

9. Shen MH, Zhang CB, Zhang JH, et al. Electroacupuncture attenuates cerebral ischemia and reperfusion injury in middle cerebral artery occlusion of rat via modulation of apoptosis, inflammation, oxidative stress, and excitotoxicity. Evid Based Complement Alternat Med. 2016;2016:9438650. doi:10.1155/2016/9438650

10. Liu L, Zhang Q, Xie HY, et al. Differences in post-ischemic motor recovery and angiogenesis of MCAO rats following electroacupuncture at different acupoints. Curr Neurovasc Res. 2020;17(1):71-78. doi:10.2174/1567202617666191223151553

11. Chen A, Lin Z, Lan L, et al. Electroacupuncture at the Quchi and Zusanli acupoints exerts neuroprotective role in cerebral ischemia-reperfusion injured rats via activation of the PI3K/Akt pathway. Int $\mathrm{J} \mathrm{Mol} \mathrm{Med.}$ 2012;30(4):791-796. doi:10.3892/ijmm.2012.1074

12. Zhang YJ, Li J, Huang W, et al. Effect of electroacupuncture combined with treadmill exercise on body weight and expression of PGC-1alpha Irisin and AMPK in skeletal muscle of diet-induced obesity rats. Zhen Ci Yan Jiu. 2019;44(7):476-480. doi:10.13702/ j.1000-0607.180460

13. Gu Y, Zheng $\mathrm{G}, \mathrm{Xu} \mathrm{M}$, et al. Caveolin-1 regulates nitric oxide-mediated matrix metalloproteinases activity and blood-brain barrier permeability in focal cerebral ischemia and reperfusion injury. $J$ Neurochem. 2012;120(1):147-156. doi:10.1111/j.14714159.2011.07542.x

14. Chen CC, Yang CL, Chang CP. An innovative running wheel-based mechanism for improved rat training performance. $J$ Vis Exp. 2016;115. doi:10.3791/54354

15. Metz GA, Whishaw IQ. The ladder rung walking task: a scoring system and its practical application. J Vis Exp. 2009;28. doi:10.3791/ 1204

16. Deacon RM. Measuring motor coordination in mice. $J$ Vis Exp. 2013;75:e2609.

17. Xu H, Mu S, Qin W. Microglia TREM2 is required for electroacupuncture to attenuate neuroinflammation in focal cerebral ischemia/ reperfusion rats. Biochem Biophys Res Commun. 2018;503 (4):3225-3234. doi:10.1016/j.bbrc.2018.08.130

18. Farmer SR, Phimister EG. Boning up on irisin. $N$ Engl $J$ Med. 2019;380(15):1480-1482. doi:10.1056/NEJMcibr1900041

19. Henningsen J, Rigbolt KTG, Blagoev B, et al. Dynamics of the skeletal muscle secretome during myoblast differentiation. Mol Cell Proteomics. 2010;9(11):2482-2496. doi:10.1074/mcp.M110.002113

20. Korta P, Pochec E, Mazur-Bialy A. Irisin as a multifunctional protein: implications for health and certain diseases. Medicina. 2019;55:8. doi:10.3390/medicina55080485

21. Huh JY, Panagiotou G, Mougios V, et al. FNDC5 and irisin in humans: I. Predictors of circulating concentrations in serum and plasma and II. mRNA expression and circulating concentrations in response to weight loss and exercise. Metabolism. 2012;61 (12):1725-1738. doi:10.1016/j.metabol.2012.09.002

22. Chen N, Li Q, Liu J, et al. Irisin, an exercise-induced myokine as a metabolic regulator: an updated narrative review. Diabetes Metab Res Rev. 2016;32(1):51-59. doi:10.1002/dmrr.2660

23. Wrann CD, White JP, Salogiannnis J, et al. Exercise induces hippocampal BDNF through a PGC-1alpha/FNDC5 pathway. Cell Metab. 2013;18(5):649-659. doi:10.1016/j.cmet.2013.09.008 
24. Gao H, Guo J, Zhao P, et al. Influences of electroacupuncture on the expression of insulin-like growth factor-1 following, focal cerebral ischemia in monkeys. Acupunct Electrother Res. 2006;31(3-4):259-272. doi: $10.3727 / 036012906815844247$

25. Zhong S, Li Z, Huan L, et al. Neurochemical mechanism of electroacupuncture: anti-injury effect on cerebral function after focal cerebral ischemia in rats. Evid Based Complement Alternat Med. 2009;6 (1):51-56. doi:10.1093/ecam/nem062

26. Kang KA, Shin ES, Hur J, et al. Acupuncture attenuates neuronal cell death in middle cerebral artery occlusion model of focal ischemia. Neurol Res. 2010;32(Suppl 1):84-87. doi:10.1179/ 016164109X12537002794246

27. Si QM, Wu GC, Cao XD. Effects of electroacupuncture on acute cerebral infarction. Acupunct Electrother Res. 1998;23(2):117-124. doi: $10.3727 / 036012998816356562$

28. Han JS. Acupuncture analgesia: areas of consensus and controversy. Pain. 2011;152(3 Suppl):S41-S48. doi:10.1016/j.pain.2010.10.012
29. Pan B, Castro-Lopes JM, Coimbra A. C-fos expression in the hypothalamo-pituitary system induced by electroacupuncture or noxious stimulation. Neuroreport. 1994;5(13):1649-1652. doi:10.1097/ 00001756-199408150-00027

30. Bostrom P, Wu J, Jedrychowski MP, et al. A PGC1-alpha-dependent myokine that drives brown-fat-like development of white fat and thermogenesis. Nature. 2012;481(7382):463-468. doi:10.1038/ nature 10777

31. Ferrer-Martinez A, Ruiz-Lozano P, Chien KR. Mouse PeP: a novel peroxisomal protein linked to myoblast differentiation and development. Dev Dyn. 2002;224(2):154-167. doi:10.1002/ dvdy. 10099

32. Ostadsharif M, Ghaedi K, Hossein NM, et al. The expression of peroxisomal protein transcripts increased by retinoic acid during neural differentiation. Differentiation. 2011;81(2):127-132. doi:10.1016/j.diff.2010.11.003

\section{Publish your work in this journal}

Neuropsychiatric Disease and Treatment is an international, peerreviewed journal of clinical therapeutics and pharmacology focusing on concise rapid reporting of clinical or pre-clinical studies on a range of neuropsychiatric and neurological disorders. This journal is indexed on PubMed Central, the 'PsycINFO' database and CAS, and is the official journal of The International Neuropsychiatric Association (INA). The manuscript management system is completely online and includes a very quick and fair peer-review system, which is all easy to use. Visit http://www.dovepress.com/testimonials.php to read real quotes from published authors. 